\title{
The role of health managers in community prevention and control of Corona Virus Disease 2019
}

\author{
Yuan Fahu ${ }^{1, \mathrm{a}}$, Chen Jiangyuan ${ }^{1}$, Chen Xiaoqing*1,b ${ }^{\text {, }}$ Chen Zhongqiang ${ }^{1, \mathrm{c}}$, Zhai Ying ${ }^{1, \mathrm{~d}}$, Ba Ruiqi ${ }^{1, \mathrm{e}}$ \\ ${ }^{1}$ School of Medicine, Jianghan University, Wuhan, China
}

\begin{abstract}
Since the outbreak of Corona Virus Disease 2019 (COVID-19) in December 2019, more than 26 million people have been infected. Urban and rural communities are the first line of defense for epidemic prevention and control. As an effective means of disease prevention and control at the community level, health management is of great significance in how to give full play to the professional advantages of health managers at present, and in combination with the current urgent situation of lack of specific drugs and vaccines, to maintain the health and social stability of the community population to the maximum extent. By assessing and intervening the health risk factors of COVID-19, the health manager can help the community effectively control the source of covid-19 infection, cut off the transmission route of COVID-19, effectively protect the vulnerable population in the community, and thus delay the spread and spread of COVID-19.
\end{abstract}

\section{INTRODUCTION}

Coronavirus Disease 2019 (COVID-19) refers to the pneumonia caused by novel Coronavirus 2019 (2019nCoV, also shortened to SARS-CoV-2) infection[1]. Since December 2019, more than 26 million confirmed cases of COVID-19 have been reported globally, and nearly 800,000 people have died. The situation of epidemic prevention and control is grim [2]. In his speech at the meeting on COVID-19 prevention and control and the deployment of economic and social development, Chinese President Xi Jinping pointed out that the two keys to the prevention and control of COVID-19 in urban and rural communities and the treatment of patients should be closely focused, the spread and export of COVID-19 should be resolutely curbed, and the grassroots, especially the community, should be substantially enriched[3]. As an important force in community health management, how health managers play a maximum role in this COVID-19 epidemic and maintain the health of the community population will have a huge impact on the effectiveness of community prevention and control.

\section{A. Pathogenic characteristics of novel Coronavirus}

Coronavirus is an RNA virus with a linear single plus strand genome, it belongs to the family Coronavirus (Nidovirales), a large family of viruses that are widespread in nature, in the systematic classification[4]. Coronavirus can be divided into the alpha, beta, gamma, delta four genera, 2019-nCoV belongs to the beta of the genus Coronavirus. 2019-nCoV is enveloped, the particles are round or elliptic, and the diameter is $60-140 \mathrm{~nm}$. The genetic characteristics of 2019-nCoV are significantly different from those of SARS-RCoV and MerSR-CoV of the same genus. After isolation and culture in vitro, the 2019-nCoV could be found in human respiratory epithelial cells within 96 hours or so to be sensitive to ultraviolet light and heat, and could be effectively inactivated by $75 \%$ ethanol, chlorine-containing disinfectant, and peracetic acid.

\section{B. Epidemiological characteristics of COVID-19}

COVID-19 infection sources are mainly pneumonia patients infected with 2019-nCoV, and the main transmission routes are respiratory droplets and close contact. Aerosol transmission is possible when exposed to aerosols in a relatively closed environment for a long time. Susceptible people have no obvious characteristics and are generally susceptible.

\section{Clinical features of COVID-19}

COVID - 19 of the incubation period is 1 to 14 days, fever, fatigue, dry cough as the main performance, a small number of patients with nasal congestion, sore throat, diarrhoea and muscle pain and other symptoms, patients with heavy after a week in more difficulty breathing and/or hypoxemia, serious rapid progress for the acute respiratory distress syndrome, sepsis, shock, difficult to correct metabolic acidosis, the blood coagulation dysfunction and multiple organ failure[5]. It should be noted that the course of the disease in severe and critical patients may be moderate to low fever, or even no obvious fever; Patients with mild symptoms showed only low fever, mild fatigue, and no manifestation of pneumonia. Most patients have a good prognosis, children have relatively mild symptoms, a small number of patients are critically ill, and the elderly and those with chronic underlying diseases have a poor prognosis.

\section{HEALTH MANAGER AND COMMUNITY HEALTH MANAGEMENT PHILOSOPHY}

Health management division is engaged in the monitoring analysis of individual or group health

\footnotetext{
ayuanfh@jhun.edu.cn_*b Corresponding author: chenxqing@jhun.edu.cn, c563132816@qq.com, d359393945@qq.com 
evaluation and health advisory guidance and intervention of professional health management set nutritionists psychological consultant, a medical doctor preventive medicine doctor health education experts medical information management and other functions in one, in order to improve the health consciousness of the whole nation and physical quality as the goal, is the comprehensive construction well-off society's important force in health management concept originated in the United States, its connotation is based on the theory and methods of management, in view of the individual or group health to conduct a comprehensive monitoring and evaluation[6].

Provide health advice and guidance, as well as risk factors for health intervention health management into the whole process of risk (found) to collect health information to assess health risk (know) health behavior intervention (solve risk) three parts in the traditional community health service through introduction of the concept of health management methods and technical means, the two promote each other coordinated development, health management and community health services in the integration of community health management, serve the community residents health together.

The traditional thinking of prevention and control of newly emerging acute respiratory infectious diseases is to use traditional biological means for prevention and control, mainly with vaccines and antiviral drugs[7]. However, this method is obviously unknown. Vaccination is an effective method for the prevention of new acute respiratory infectious diseases, and it is also the most effective and targeted technical means at present. However, it can only be used to protect vulnerable groups after the occurrence of the disease and after a period of time of successful development when the disease has caused considerable impact.Therefore, it is impossible for people to find specific vaccines to control the spread of diseases immediately after the emergence of new infectious diseases. Due to the limitations of science and technology, human's understanding of the future change rules of diseases is still full of variables and uncertainties, including the transmissibility, variability and pathogenicity of pathogens[8]. Therefore, the development of specific drugs will cost a lot of time and money, and after the development of drugs, due to the limitation of production capacity, side effects and pathogen resistance and other problems have restricted the group defense effect of drugs.

New thinking and advantages of health management in the prevention and control of emerging acute respiratory infectious diseases.The experience that mankind accumulates in fight with infectious disease for a long time makes clear, the happening of any human infectious disease and epidemic all depend on 3 respects factor, namely infect source, transmission route and susceptible crowd.Effective prevention and control of infectious diseases can be achieved by reducing or eliminating health risk factors related to these three aspects through various scientific methods and technical means.Health management as a kind of brand-new concept of disease prevention and control of health promotion, through the intervention of health risk factors, the intervention and the source of infection, route of transmission, and vulnerable groups of the health risk factors associated with three aspects obtains, seeking to achieve a comprehensive control infection effectively, to cut off the route of transmission and protection of vulnerable groups, the purpose of reducing the spread of the epidemic and spread.The great superiority of health management in the prevention and control of emerging acute respiratory infectious diseases is considered as the most feasible measure to reduce or delay the emerging acute respiratory infectious diseases pandemic before the emergence of effective vaccines and specific drugs.

Of community is the basic unit of the city, carrying the vast majority of residents' daily life of the community of dense flow, logistics, information flow determines its people more vulnerable to a variety of new acute respiratory infectious diseases, in the face of the sudden outbreak of COVID - 19, community residents with high susceptibility, generally due to COVID - 19 mainly spread through the respiratory tract, the scope and speed of the spread of the epidemic in the community may multiply.Therefore, improving community prevention and control capacity is the key to effectively deal with the occurrence and prevalence of new acute respiratory infectious diseases in the community.

Community people due to the lack of immunity against new acute respiratory infectious diseases, everyone is disease of natural exposure, once the outbreak was introduced into the community, in the face of the community of high-risk groups and vulnerable groups, the outbreak will penetrate, form widely spread and spread, health management through dynamic disease surveillance can timely found that residents of flu-like symptoms, timely inspection samples, isolation and take necessary treatment measures in time after confirmed.Thus reducing or delaying the wider spread of the epidemic will buy valuable time for the development of specific diagnostic reagents, vaccines and specific drugs at the national level.It also prepares the public psychologically for the possibility of a larger outbreak.

Outbreak epidemic period, because of its highly pathogenic, with community residents do not understand disease, widespread panic psychology, which makes them focus on their health than ever more intense, the prevention and control of epidemic diseases progress information demand is more urgent, health management division give full play to the huge advantage in providing health related information, through health education and health promotion will lead to disease prevention and control of relevant knowledge and skill to the community residents, emphasis on individual performance of the responsibility for their own health, make them consciously develop healthy and civilized way of life, and maintain a psychological balance to better maintain and promote their own health.

In an emergency, a community is a community with a Shared future for all residents. The prevention and control of coVID-19 is related to the vital interests of everyone. The infection of any individual will pose a serious threat to the health of the entire community.Health management should be carried out in the community and all available resources should be mobilized to enhance residents' sense of ownership in the prevention and control of new acute respiratory infectious diseases, enhance mutual 
understanding and support among residents and strengthen community cohesion, thus building a strong barrier for the prevention and control of new acute respiratory infectious diseases.

\section{HIGHLIGHTS OF THE WORK OF COMMUNITY HEALTH MANAGERS IN THE COVID-19 EPIDEMIC}

The new acute respiratory infectious diseases are characterized by uncertainty, abruptness and insufficient cognition in the initial stage, which determines that prevention should be the priority and mass prevention and mass control should be the primary choice for prevention and control, so as to delay or reduce the spread of the epidemic in the community to the greatest extent.Health management is an important means and way to implement the policy of putting prevention first and realize mass prevention and mass control. Through formulating and implementing strategies and measures of prevention, control, treatment and effective response, it can stop the epidemic of diseases and protect the health of community population.

A. Carry out health screening and establish health records of community residents

Through household surveys online survey physical form of community residents of health related information collection, to the health of the community residents to establish standardized file standardized residents' health records help to community health service center is a comprehensive hospital center for disease control and prevention institutions such as the fast continuously residents health information exchange and sharing, strengthen mutual cooperation, to implement the dynamic management and the real-time monitoring of residents' health.

Based on the epidemiological characteristics of COVID-19, high-risk groups, such as pregnant women, the elderly, patients with chronic underlying diseases and long-term use of immunosuppressive agents, will be selected from the general population to make full use of the perfect standardized health records, and graded management will be implemented to provide health consultation and guidance services.

\section{B. Full implementation of health education activities}

Health education is the education activity organized by planned system, make the community residents consciously adopt healthy behavior and way of life in online media,such as WeChat public platform and SinaWeibo, brochures, and other forms of universal COVID19 prevention and control knowledge, improve community residents of COVID-19 the cognitive level of harm and prevention knowledge, enables them to master the simple and effective means of prevention and control measures, and convert the knowledge and skills to promote health conscious action.

Keep the information communication with the community residents, educate the community residents to eliminate unnecessary panic mentality while keeping certain vigilance, guide the cultivation of good personal hygiene habits, and consciously do a good job of selfisolation.

\section{Assist in epidemiological investigation}

Once the COVID-19 outbreak, the health management division community should work closely with institution of disease prevention and control, tracking potential sources of infection, and close contacts of patients with suspected screening, when necessary to do ideological work, and to protect the screening work screening to contain the outbreak, as early as possible to prevent the spread of disease and contagion. The epidemic focus screening, isolation have fever residents under medical observation, to prevent imported cases that may occur in the community, causing the spread of disease, for close contacts, should be followed up every day, guide to do a good job of disinfection protection, timely and necessary psychological intervention, closely tracking, and take the necessary measures in time.

\section{Strict management of new and suspected COVID-19} cases

Confirmed cases of COVID-19 should be subjected to mandatory isolation and treatment in accordance with the law and reported in a timely manner.Of concentration of suspected cases and close contacts medical observation or observation that occupy the home, the first clinical doctors on the daily follow-up, closely tracking the progress of the disease and take necessary measures in time, cure need both physical treatment of the disease, need more psychological persuation. COVID - 19 patients' psychological burden than patients with other chronic diseases, and is much bigger than the burden of the disease itself, they face isolation, the situation of discrimination, alone, fear, pressure, so to provide necessary psychological support is indispensable, consciously abide by social morality education patients at the same time, attention and control their own behavior.

\section{E. Health guidance for discharged patients}

For COVID-19, which is prone to social panic, although patients are discharged from hospital after treatment and recovery, patients and their families remain concerned to varying degrees about the recurrence and transmission of the disease to others, as well as the potential for discrimination. Community health management division should follow up the follow-up and provide the necessary supportive care, in particular, do a good job for the psychological guidance of the survivors and their families, close communication, and for them to give timely and scientific solutions, eliminate their anxiety and other bad feelings, make them a correct understanding and treatment of diseases, establish confidence to conquer the disease. 


\section{CONSTRUCTION OF A COMPREHENSIVE PREVENTION AND CONTROL MODEL FOR COVID-19}

SARS and H1N1 influenza prevention and control work of practice has shown that: in the face of sudden acute respiratory infectious disease outbreaks, build a comprehensive prevention and control system, the importance of the construction of this system will change the disorder of prevention and control to improve the efficiency of the prevention and control work in an orderly way, and save health resources, reduce social costs, and conducive to maintaining social stability.

Guided by the concept of health management of community COVID-19 of the prevention and control of the construction of comprehensive prevention and control system of variable disorderly to orderly, and implement the thought of prevention prevention \& treatment combination, achieved the combination of long-term management and emergency disposal, realized the unity of professional prevention and control and monitoring group control, built a control COVID-19 steel wall, which is beneficial to outbreak occurs, improve the work efficiency to save health resources, reduce social costs,In addition, the establishment of the comprehensive prevention and control system also provides new ideas for the effective prevention and control of other emerging infectious diseases.

COVID-19 with uncertainty, sudden, and the characteristics of cognitive deficiencies decided the prevention, control group control become the primary choice of prevention and control work, in order to minimize or delay spread of the spread of epidemic in the community and health management policy is to carry out prevention work, realize the important means and way of control group control, through the formulation and implementation of control and prevention, cure and effectively cope with the intervention strategies and measures of all possible infection of severe acute respiratory infectious diseases, new health risk factors, to block the -epidemic, the purpose of the security community crowd health.

\section{CONCLUTION}

At the present stage, when the incidence of COVID-19 continues to rise, it is often difficult to effectively control its incidence trend, which leads to the stable development of the whole society being affected to some extent, and at the same time, it is easy to cause social panic.So, want to better realize the prevention and control of infectious diseases, should be to the community as a unit to prevention and control work, at present, under the philosophy of health management should pay attention to build up perfect health information system, but also improve the professional quality of community health management division itself, finally also should establish health records, can also with the help of the infectious diseases prevention and control personnel, through community health centers, kindergartens, schools, and by their parents the masses through social software, timely communication, do a good job in health education, found the problem, collect, also have the effect very much to prevent these diseases,In this way, comprehensive screening of the health status of community residents can be achieved, which can effectively improve the prevention and control level of COVID-19.

\section{ACKNOWLEDGMENT}

This project is funded by the Engineering Technology Research Center for the Protection, Development and Utilization of Characteristic biological Resources in The Hanjiang River Basin of Hubei Province. This paper is one of the phased results of the open subject project (2019-06) of the Engineering Technology Research Center for the Protection, Development and Utilization of Characteristic biological resources in the Hanjiang River Basin of Hubei Province.

\section{REFERENCES}

[1] D. Don, C. Giaquinto, E. Baraldi, A. Biffi, P. Gamba, AM. Saieva et al., "COVID-19 pandemic: perspective of an Italian Tertiary Care Pediatric Center.," Healthcare (Basel, Switzerland), vol. 8, 2020.

[2] TT. Le, JP. Cramer, R. Chen, and S. Mayhew, "Evolution of the COVID-19 vaccine development landscape.," Nature reviews. Drug discovery, 2020.

[3] TC. Yeh, HC. Huang, TY. Yeh, WT. Huang, HC. Huang, YM. Chang, and W. Chen, 'Family members' concerns about relatives in long-term care facilities: Acceptance of visiting restriction policy amid the COVID-19 pandemic.," Geriatrics \& gerontology international, 2020.

[4] H. Xu, MJ. Gonzalez, L. Guo, Q. Chen, L. Zheng, P. Chen, X Cao, et al., "Knowledge, awareness, and attitudes towards COVID-19 pandemic among different populations in central China: a cross-sectional survey.," Journal of medical Internet research, 2020.

[5] D. Mendelson, M. Keane, M. Bagaric, and C. Graydon, "Legal implications of personal protective equipment use when treating patients for COVID-19 (SARS-CoV-2).," Journal of law and medicine, vol. 24, pp. 856-864, 2020.

[6] VA. Karatayev, M. Anand, and CT. Bauch, "Local lockdowns outperform global lockdown on the far side of the COVID-19 epidemic curve.," Proceedings of the National Academy of Sciences of the United States of America, 2020.

[7] J. Yan, and G. Yang, "Nursing standard of internet-based rehabilitation for patients with coronavirus disease 2019.," Journal of Central South University. Medical sciences, vol.45, pp. 513 517, 2020.

[8] AM. Buttenheim, "SARS-CoV-2 vaccine acceptance: we may need to choose our battles.,"Annals of internal medicine, 2020. 